\title{
Dynamic Modeling and Control of Electromechanical Coupling for Mechanical Elastic Energy Storage System
}

\author{
Yang Yu and Zengqiang Mi \\ State Key Laboratory of Alternate Electrical Power System with Renewable Energy Sources, North China Electric Power University, \\ Baoding 071003, China
}

Correspondence should be addressed to Yang Yu; ncepu_yy@163.com

Received 12 July 2013; Accepted 4 September 2013

Academic Editor: Michael Chen

Copyright (C) 2013 Y. Yu and Z. Mi. This is an open access article distributed under the Creative Commons Attribution License, which permits unrestricted use, distribution, and reproduction in any medium, provided the original work is properly cited.

The structural scheme of mechanical elastic energy storage (MEES) system served by permanent magnet synchronous motor (PMSM) and bidirectional converters is designed. The aim of the research is to model and control the complex electromechanical system. The mechanical device of the complex system is considered as a node in generalized coordinate system, the terse nonlinear dynamic model of electromechanical coupling for the electromechanical system is constructed through Lagrange-Maxwell energy method, and the detailed deduction of the mathematical model is presented in the paper. The theory of direct feedback linearization (DFL) is applied to decouple the nonlinear dynamic model and convert the developed model from nonlinear to linear. The optimal control theory is utilized to accomplish speed tracking control for the linearized system. The simulation results in three different cases show that the proposed nonlinear dynamic model of MEES system is correct; the designed algorithm has a better control performance in contrast with the conventional PI control.

\section{Introduction}

Power balance is a basis for stable operation of power system $[1,2]$, which is frequently disturbed by some factors such as faults and intermittent power generations. These threatening behaviors may lead to oscillation between the generating units and the interconnected systems, and even worse, which probably cause serious large-scale blackout in power system [3]. Currently, one of the principal methods solving the power imbalance is to reserve adequate spare capacity for the system, which not only increases the investment, but also reduces the utilization of electrical equipments. With the development of energy storage (ES) technologies, a new way of utilizing energy storage technology to solve the power imbalance has been paid more and more attentions $[4,5]$. From state-of-theart technologies of ES, a great variety of the technologies have been developed [6-11]. However, from a viewpoint of power system operation, the technologies of ES at least need to have the following features: large capacity, fast power response speed, high efficiency, long service life, low operation cost and environmental friendship, and so forth. In terms of these requests, most of ES technologies are still immature except pumped-storage. A new method utilizing spiral springs as ES medium is proposed in reference [12], which is called mechanical elastic energy storage (MEES); some of the basic characteristics for the technology are discussed in [13]. Due to the benefits of small size, low loss, and high efficiency [14], permanent magnet synchronous motor (PMSM) is chosen as the servomotor for the MEES system. Thus, the MEES system, which is integrated by the mechanical device (spiral springs and the correspondingly coating shell), the electrical installation (PMSM), and relevant control information elements, is a typically complex nonlinear system of electromechanical coupling. Parameter coupling, which is induced between electromagnetic parameters of PMSM and mechanical parameters of spiral springs, may affect the dynamic performance of the system. The preexisting studies have shown that revealing the law of electromechanical interaction from the point of view of energy is an effective solution to research dynamic characteristics of complex electromechanical system. Lagrange-Maxwell equation of flywheel energy storage is deduced in reference [15] by 
calculation of integral of magnetic density in the area of magnetic field.

The modeling and control of complex dynamic systems are the rapidly emerging researching topics [16], especially for the nonlinearities in the systems [17]. The paper concentrates on modeling and decoupling control of complex PMSM based MEES system in detail. The mechanical device is taken into account in the analysis of electromechanical coupling, which can make the research become closer to the reality. Especially for the mechanical spiral springs with a big quality and large inertia, neglecting their influence will make the results inaccurate, even unavailable. The overall aim of the paper is to model and control the complex electromechanical system based on the characteristics of mechanical spiral springs in order to improve the operating stability of the system; in particular, the main innovative points of the research are represented as follows:

(a) previous researches mainly focus on the motor ontology for the electromechanical issue; the difference in the research is to consider the mechanical device as a node in generalized coordinate system; the terse nonlinear dynamic model for a complex electromechanical system is deduced and constructed through Lagrange-Maxwell energy method by the principle of electromechanical coupling.

(b) the generalized coordinates of MEES system are determined. The functions of the kinetic energy, magnetic energy, the potential energy, and the energy dissipation of the whole system are obtained, and the differential equations with electromechanical parameters of MEES system are established by applying the extended Lagrange-Maxwell equation.

(c) the theory of direct feedback linearization (DFL) is applied to decouple the nonlinear model and convert the developed model for MEES system from nonlinear to linear. An optimal linear controller is designed to accomplish the speed tracking control for the linearized model.

The paper is organized as the following: it starts with introductions to the ES technologies and the electromechanical characteristics of MEES system in Section 1. The constitution and operating principles of the MEES system are described in Section 2. Mathematical modeling of electromechanical coupling for the complex MEES system is deduced in Section 3. In Section 4, DFL based decoupling control of the dynamic model is proposed, and an optimal linear controller is designed to accomplish the speed tracking control for the linearized model. Section 5 gives the results of numerical simulation. Finally, the conclusions are summarized in Section 6.

\section{Preliminaries and Problem Description}

2.1. Constitution of MEES System. In order to give the readers a better understanding of the operating principle of the research object, the composition scheme of MEES system served by PMSM and bidirectional converters is described in Figure 1.
PMSM based MEES system consists of several elements: ES box (multiple spiral springs wrapped up in it), gear box, PMSM, bidirectional converters, breaker, and controller. Therefore, the electromechanical coupling is caused by the interaction between electromagnetic parameters of PMSM and mechanical parameters of spiral springs. A MEES power station can be built by getting tens or even more units together. Through a central controller, the units in the power station can be controlled orderly to realize the goal of largescale energy storage and power generation. It is important to note that a linkage form structure for ES box is proposed in order to increase the energy storage capacity; for details see [13].

2.2. Operating Principle of MEES System. The basic operation principle of the MEES system is concerned with two processes: energy storage and power generation. The load or drive source of MEES system in energy storage or power generation is spiral springs in ES box. In the process of energy storage, PMSM, which is driven by power grid, tightens the springs through gear box. Then, the electric energy is stored in the form of elastic deformation energy. Once the unit receives a signal of releasing energy, the tightened springs start to release energy and drive the motor to generate electricity.

\section{Modeling of Electromechanical Coupling for Complex MEES System}

3.1. Physical Analysis. The operating rotation speed of the spindle in elastic ES box in the process of energy storage or power generation is required to run basically stable owing to the large size and the big inertia of ES box. As mentioned above, the elastic ES box can be considered as an output load of PMSM in ES. Hence, supposing that the rotation speed of the shaft in ES box is a constant, the torque calculation formula of ES box in ES process can be given by

$$
T=\frac{E B H^{3} \pi}{6 L} \omega t+T_{0},
$$

where $T$ and $T_{0}$ denote the operating torque and initial torque, $E, B, H$, and $L$ are the modulus of elasticity, width, thickness, and length of spring material, and $t$ denotes the time. It should be noted that $T$ must be ensured between the maximum torque and minimum torque. Additionally, it should be compatible with the electromagnetic torque of PMSM. In terms of formula (1), the characteristic curve for ES box in ES can be shown in Figure 2.

The servomotor of PMSM consists of two parts, stator and rotor. When three-phase alternating current (AC) with mutual phase difference of $120^{\circ}$ is flowed into the stator windings of the motor, a rotating magnetic field with a uniform motion in space is induced. The rotation speed of the magnetic field is related to the frequency of sinusoidal wave in stator windings. The driven torque, which is produced by the interaction between the rotating magnetic field of stator and permanent magnetic field of rotor, makes the rotor rotate and achieves the conversion of electric energy to elastic energy. 


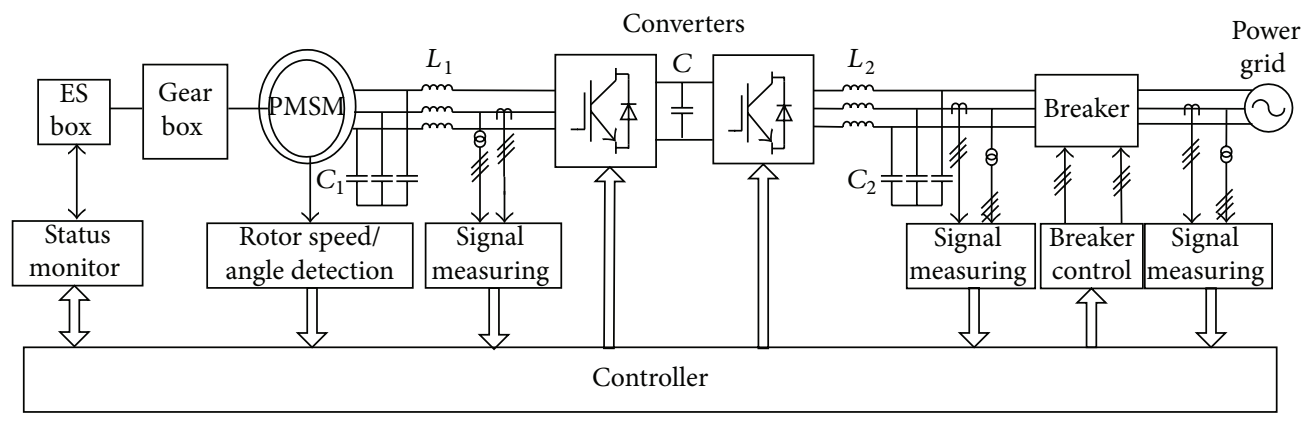

FIGURE 1: The composition scheme of MEES system served by PMSM and bidirectional converters.

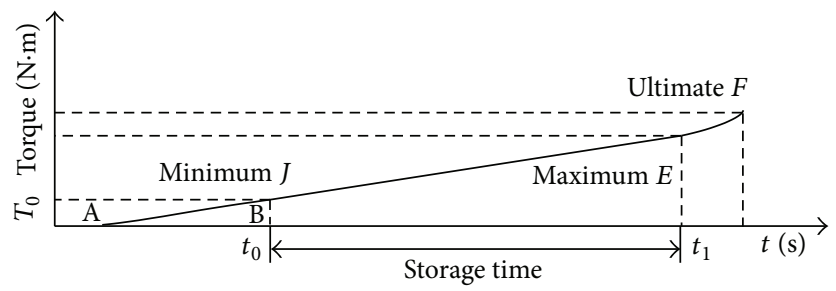

Figure 2: Characteristics curve for ES box in ES.

In the process of ES, the torque of the springs raises with the operation of the system. The power angle between the axis of stator and rotor becomes larger, which augments the magnetic torque to balance the increasing torque of springs. With the rising of energy stored in ES box, the input power and the stator current increase accordingly. Simultaneously, the armature reaction causes the growth of flux linkage of air gap and back electromotive force (e.m.f) of stator and compels the stator voltage to rise. In other words, the interaction between the electromagnetic parameters of electrical system and the mechanical parameters of mechanical system composes the electromechanical coupling in complex MEES system.

3.2. Mathematical Modeling. Before modeling the system of electromechanical coupling, six assumptions are described as follows:

(a) the saturation of the core is neglected,

(b) the losses of eddy and hysteresis are ignored,

(c) the distribution of air gap is uniform,

(d) the self-inductance and mutual inductance among the windings are independent of the position of the rotor,

(e) the damping winding in the rotor and the damping effect of permanent magnet are both neglected,

(f) back e.m.f. maintains the sinusoidal variation.

Based on the principle of electromechanical coupling dynamics, Lagrange-Maxwell energy method is applied to construct the dynamic mathematical model of MEES system as follows.

(1) Determining Generalized Coordinates of the System. Owing to the large size, big quality, and high inertia of the mechanical spiral springs, neglecting their influence will make the results inaccurate, even unavailable. Hence, the mechanical device is taken into account and considered as a node in generalized coordinate system. Six generalized coordinates shown in Table 1 are constructed for the MEES system, four of which belong to electromagnetic system $(k=1,2,3,4)$, and the remaining two are mechanical system $(j=1,2)$. The parametric descriptions of six generalized coordinates shown in Table 1 are as follows: $i_{a}, i_{b}$, and $i_{c}$ are three-phase AC flowed in stator, $u_{a}, u_{b}$, and $u_{c}$ indicate three-phase voltage applied in stator, $\psi_{f}$ represents the magnetic flux of permanent magnet in rotor, $\vartheta_{1}$ and $\vartheta_{2}$ are the angular displacement of rotor and the spindle of ES box, respectively, $\Omega_{1}$ and $\Omega_{2}$ are the mechanical angular velocity of rotor and spindle of ES box, respectively, and $T_{g}$ and $T_{L}$ represent the torque of gear and load. $T_{L}$ is equal to the torque $T$ of the springs in the ES box in energy storage.

(2) Finding Out the Energy Relationship for the MEES System. The kinetic energy of the MEES system $W_{k}$ can be given as

$$
\begin{aligned}
W_{k} & =\frac{1}{2} J_{1} \Omega_{1}^{2}+\frac{1}{2} J_{e} \Omega_{1}^{2}+\frac{1}{2} J_{\mathrm{sp}} \Omega_{2}^{2} \\
& =\frac{1}{2} J_{1} \Omega_{1}^{2}+\frac{1}{2} J_{e} \Omega_{1}^{2}+\frac{1}{2} J_{\mathrm{sp}}\left(\frac{\Omega_{1}}{i}\right)^{2},
\end{aligned}
$$

where $J_{1}$ is the moment of inertia of PMSM, $J_{e}$ and $J_{\mathrm{sp}}$ are the equivalent moment of inertia of the gear and ES box with respect to input shaft, and $i$ is the transformation ratio of gear.

For the MEES system, the magnetic energy $W_{m}$, which is jointly produced by the stator current, permanent magnet of rotor, and interaction with each other, consists of three parts. 
TABLE 1: Six generalized coordinates for MEES system.

\begin{tabular}{|c|c|c|c|c|c|c|}
\hline \multirow{3}{*}{ Generalized coordinates } & \multicolumn{4}{|c|}{ Electromagnetic system } & \multicolumn{2}{|c|}{ Mechanical system } \\
\hline & & Stator & & Rotor & Gear & ES box \\
\hline & $k=1$ & $k=2$ & $k=3$ & $k=4$ & $j=1$ & $j=2$ \\
\hline$e_{k}\left(q_{j}\right)$ & - & - & - & $\psi_{f}$ & $\theta_{1}$ & $\theta_{2}$ \\
\hline$\dot{e}_{k}\left(\dot{q}_{j}\right)$ & $i_{a}$ & $i_{b}$ & $i_{c}$ & - & $\Omega_{1}$ & $\Omega_{2}$ \\
\hline$U_{k}\left(Q_{j}\right)$ & $u_{a}$ & $u_{b}$ & $u_{c}$ & - & $-T_{g}$ & $-T_{L}$ \\
\hline
\end{tabular}

The calculation of the magnetic energy $W_{m 1}$ produced by the current of stator is given as

$$
W_{m 1}=\frac{1}{2} L_{a} i_{a}^{2}+\frac{1}{2} L_{b} i_{b}^{2}+\frac{1}{2} L_{c} i_{c}^{2}+M i_{a} i_{b}+M i_{b} i_{c}+M i_{c} i_{a}
$$

where $L$ and $M$ indicate the self-inductance and mutual inductance of three-phase windings in stator, for an exteriorgibbose PMSM, $M_{a a}=M_{b b}=M_{c c}=M_{a b}=M_{b c}=M_{c a}=$ $M, L_{a}=L_{b}=L_{c}$.

For the distributed winding with a phase belt of $60^{\circ}$, due to the number $q$ of the slots for per pole per phase is often more than two, the magnetic energy of air gap $W_{m 2}$ can be considered not changed with the rotation of rotor, namely, the magnetic energy produced by permanent magnet of rotor is a constant, $W_{m 2}=C_{1}\left(C_{1}\right.$ is a constant $)$.

$W_{m 12}$ is the magnetic energy produced by the interaction between the stator current and stator flux generated by the rotor; the calculation formula of $W_{m 12}$ can be written as

$$
\begin{aligned}
W_{m 12}= & i_{a} \psi_{f} \cos \theta_{1}+i_{b} \psi_{f} \cos \left(\theta_{1}-\frac{2}{3} \pi\right) \\
& +i_{c} \psi_{f} \cos \left(\theta_{1}+\frac{2}{3} \pi\right) .
\end{aligned}
$$

The potential energy of the system $V$ is given by

$$
V=\frac{1}{2} \alpha_{\mathrm{sp}} \theta_{2}^{2}
$$

where $\alpha_{\text {sp }}$ is a torsion constant of the springs.

The Lagrange function can be written as

$$
\begin{aligned}
L= & W_{k}+W_{m}-V=\frac{1}{2} J_{1} \Omega_{1}^{2}+\frac{1}{2} J_{e} \Omega_{1}^{2}+\frac{1}{2} J_{\mathrm{sp}} \Omega_{2}^{2} \\
& +\frac{1}{2} L_{a} i_{a}^{2}+\frac{1}{2} L_{b} i_{b}^{2}+\frac{1}{2} L_{c} i_{c}^{2} \\
& +M i_{a} i_{b}+M i_{b} i_{c}+M i_{c} i_{a}+C_{1} \\
& +i_{a} \psi_{f} \cos \theta_{1}+i_{b} \psi_{f} \cos \left(\theta_{1}-\frac{2}{3} \pi\right) \\
& +i_{b} \psi_{f} \cos \left(\theta_{1}+\frac{2}{3} \pi\right)-\frac{1}{2} \alpha_{\mathrm{sp}} \theta_{2}^{2} .
\end{aligned}
$$

The dissipation function of magnetic system $F_{e}$ can be expressed as

$$
F_{e}=\frac{1}{2} R_{a} i_{a}^{2}+\frac{1}{2} R_{b} i_{b}^{2}+\frac{1}{2} R_{c} i_{c}^{2}
$$

where $R_{a}, R_{b}$, and $R_{c}$ indicate three-phase resistance of the stator.

The dissipation function of mechanical system $F_{m}$ can be written as

$$
F_{m}=\frac{1}{2}\left(B_{1}+B_{e}\right) \Omega_{1}^{2}+\frac{1}{2} B_{\mathrm{sp}}\left(\frac{\Omega_{1}}{i}\right)^{2},
$$

where $B_{1}, B_{e}$, and $B_{\mathrm{sp}}$ are the equivalent viscous damping coefficients of the motor, the gear, and the spring, respectively.

Then, the dissipation function of the whole system $F$ can be given as

$$
\begin{aligned}
F= & F_{e}+F_{m}=\frac{1}{2} R_{a} i_{a}^{2}+\frac{1}{2} R_{b} i_{b}^{2}+\frac{1}{2} R_{c} i_{c}^{2} \\
& +\frac{1}{2}\left(B_{1}+B_{e}\right) \Omega_{1}^{2}+\frac{1}{2} B_{\mathrm{sp}}\left(\frac{\Omega_{1}}{i}\right)^{2} .
\end{aligned}
$$

Nonconservative generalized force of mechanical system $(j=1,2)$ can be expressed as

$$
\begin{array}{ll}
Q_{1}=-T_{g}, & j=1, \\
Q_{2}=-T_{L}, & j=2 .
\end{array}
$$

External e.m.f. of electromagnetic system is given by

$$
U_{k}=-u_{k}
$$

The Lagrange-Maxwell equation of MEES system can be expressed as

$$
\begin{aligned}
& \frac{d}{d t}\left(\frac{\partial L}{\partial \dot{e}_{k}}\right)-\frac{\partial L}{\partial e_{k}}+\frac{\partial F}{\partial \dot{e}_{k}}=U_{k} \\
& \frac{d}{d t}\left(\frac{\partial L}{\partial \dot{q}_{j}}\right)-\frac{\partial L}{\partial q_{j}}+\frac{\partial F}{\partial \dot{q}_{j}}=Q_{j}
\end{aligned}
$$

For electromagnetic system, firstly, the stator winding of A-phase $(k=1)$ is considered as

$$
\begin{gathered}
\frac{\partial L}{\partial e_{1}}=0 \\
\frac{\partial L}{\partial \dot{e}_{1}}=\frac{\partial L}{\partial i_{a}}=L i_{a}+M i_{b}+M i_{c}+\psi_{f} \cos \theta_{1}
\end{gathered}
$$




$$
\begin{gathered}
\frac{d}{d t}\left(\frac{\partial L}{\partial \dot{e}_{1}}\right) \longrightarrow \frac{d}{d t}\left(\frac{\partial L}{\partial i_{a}}\right)=L \frac{d i_{a}}{d t}+M \frac{d i_{b}}{d t}+M \frac{d i_{c}}{d t} \\
+\frac{d\left(\psi_{f} \cos \theta_{1}\right)}{d t}, \\
\frac{\partial F}{\partial \dot{e}_{1}} \longrightarrow \frac{\partial F}{\partial i_{a}}=R_{a} i_{a} \\
U_{1}=\frac{d}{d t}\left(\frac{\partial L}{\partial \dot{e}_{1}}\right)-\frac{\partial L}{\partial e_{1}}+\frac{\partial F}{\partial \dot{e}_{1}} \\
u_{a}=U_{1} ;
\end{gathered}
$$

the voltage equation of stator winding of A-phase can be written as

$$
\begin{aligned}
u_{a}= & U_{1}=\frac{d}{d t}\left(\frac{\partial L}{\partial \dot{e}_{1}}\right)-\frac{\partial L}{\partial e_{1}}+\frac{\partial F}{\partial \dot{e}_{1}} \\
= & L \frac{d i_{a}}{d t}+M \frac{d i_{b}}{d t}+M \frac{d i_{c}}{d t} \\
& +\frac{d\left(\psi_{f} \cos \theta_{1}\right)}{d t}+R_{a} i_{a} .
\end{aligned}
$$

Similarly, the voltage equation of stator winding of Bphase $(k=2)$ can be written as

$$
\begin{aligned}
u_{b}= & U_{2}=\frac{d}{d t}\left(\frac{\partial L}{\partial \dot{e}_{2}}\right)-\frac{\partial L}{\partial e_{2}}+\frac{\partial F}{\partial \dot{e}_{2}} \\
= & L \frac{d i_{b}}{d t}+M \frac{d i_{c}}{d t}+M \frac{d i_{a}}{d t} \\
& +\frac{d\left(\psi_{f} \cos \left(\theta_{1}-(2 / 3) \pi\right)\right)}{d t}+R_{b} i_{b} ;
\end{aligned}
$$

the voltage equation of stator winding of C-phase $(k=3)$ can be written as

$$
\begin{aligned}
u_{c}= & U_{3}=\frac{d}{d t}\left(\frac{\partial L}{\partial \dot{e}_{3}}\right)-\frac{\partial L}{\partial e_{3}}+\frac{\partial F}{\partial \dot{e}_{3}} \\
= & L \frac{d i_{c}}{d t}+M \frac{d i_{a}}{d t}+M \frac{d i_{b}}{d t} \\
& +\frac{d\left(\psi_{f} \cos \left(\theta_{1}+(2 / 3) \pi\right)\right)}{d t}+R_{c} i_{c} .
\end{aligned}
$$

For permanent magnet of rotor, the effective flux $\psi_{f}$ of permanent magnet is generally considered to be a constant, namely, $\psi_{f}=C_{2}\left(C_{2}\right.$ is a constant $)$. Thus

$$
\frac{\partial L}{\partial \psi_{f}}=0, \quad \frac{\partial L}{\partial \dot{\psi}_{f}}=0 .
$$

For mechanical system, when $j=1$ has

$$
\begin{aligned}
\frac{\partial L}{\partial q_{1}} \longrightarrow \frac{\partial L}{\partial \theta_{1}}= & -i_{a} \psi_{f} \sin \theta_{1}-i_{b} \psi_{f} \sin \left(\theta_{1}-\frac{2}{3} \pi\right) \\
& -i_{c} \psi_{f} \sin \left(\theta_{1}+\frac{2}{3} \pi\right)-\alpha_{\mathrm{sp}} \frac{\theta_{1}}{i^{2}},
\end{aligned}
$$

$$
\begin{gathered}
\frac{\partial L}{\partial \dot{q}_{1}} \longrightarrow \frac{\partial L}{\partial \Omega_{1}}=\left(J_{1}+J_{e}\right) \Omega_{1}+J_{\mathrm{sp}} \frac{\Omega_{1}}{i^{2}} \\
\frac{d}{d t}\left(\frac{\partial L}{\partial \dot{q}_{1}}\right) \longrightarrow \frac{d}{d t}\left(\frac{\partial L}{\partial \Omega_{1}}\right)=\left(J_{1}+J_{e}+\frac{J_{\mathrm{sp}}}{i^{2}}\right) \frac{d \Omega_{1}}{d t} \\
\frac{\partial F}{\partial \dot{q}_{1}} \longrightarrow \frac{\partial F}{\partial \Omega_{1}}=\left(B_{1}+B_{e}+\frac{B_{\mathrm{sp}}}{i^{2}}\right) \Omega_{1} \\
Q_{1}=\frac{d}{d t}\left(\frac{\partial L}{\partial \dot{q}_{1}}\right)-\frac{\partial L}{\partial q_{1}}+\frac{\partial F}{\partial \dot{q}_{1}} \\
Q_{1}=-T_{g}
\end{gathered}
$$

the motion equation of mechanical system $j=1$ can be expressed as

$$
\begin{aligned}
\left(J_{1}+J_{e}+\frac{J_{\mathrm{sp}}}{i^{2}}\right) \frac{d \Omega_{1}}{d t}= & -i_{a} \psi_{f} \sin \theta_{1}-i_{b} \psi_{f} \sin \left(\theta_{1}-\frac{2}{3} \pi\right) \\
& -i_{c} \psi_{f} \sin \left(\theta_{1}+\frac{2}{3} \pi\right)-\alpha_{\mathrm{sp}} \frac{\theta_{1}}{i^{2}} \\
& -\left(B_{1}+B_{e}+\frac{B_{\mathrm{sp}}}{i^{2}}\right) \Omega_{1}-T_{g} .
\end{aligned}
$$

Let $J=J_{1}+J_{e}+\left(J_{\mathrm{sp}} / i^{2}\right)$ and $B=B_{1}+B_{e}+\left(B_{\mathrm{sp}} / i^{2}\right)$; the motion equation of mechanical system $j=1$ can be rewritten as

$$
\begin{aligned}
J \frac{d \Omega_{1}}{d t}= & -i_{a} \psi_{f} \sin \theta_{1}-i_{b} \psi_{f} \sin \left(\theta_{1}-\frac{2}{3} \pi\right) \\
& -i_{c} \psi_{f} \sin \left(\theta_{1}+\frac{2}{3} \pi\right)-\alpha_{\mathrm{sp}} \frac{\theta_{1}}{i^{2}}-B \Omega_{1}-T_{g} .
\end{aligned}
$$

For mechanical system, when $j=2$ has

$$
\begin{gathered}
\frac{\partial L}{\partial q_{2}} \longrightarrow \frac{\partial L}{\partial \theta_{2}}=-i i_{a} \psi_{f} \sin \theta_{2}-i i_{b} \psi_{f} \sin \left(\theta_{2}-\frac{2}{3} \pi\right) \\
-i i_{c} \psi_{f} \sin \left(\theta_{1}+\frac{2}{3} \pi\right)-\alpha_{\mathrm{sp}} \theta_{2}, \\
\frac{\partial L}{\partial \dot{q}_{2}} \longrightarrow \frac{\partial L}{\partial \Omega_{2}}=i^{2}\left(J_{1}+J_{e}\right) \Omega_{2}+J_{\mathrm{sp}} \Omega_{2}, \\
\frac{d}{d t}\left(\frac{\partial L}{\partial \dot{q}_{2}}\right) \longrightarrow \\
\frac{\partial F}{\partial t}\left(\frac{\partial L}{\partial \Omega_{2}}\right)=\left[i^{2}\left(J_{1}+J_{e}\right)+J_{\mathrm{sp}}\right] \frac{d \Omega_{2}}{d t}, \\
\frac{\partial F}{\partial \Omega_{2}}=\left[i^{2}\left(B_{1}+B_{e}\right)+B_{\mathrm{sp}}\right] \Omega_{2}, \\
Q_{2}=\frac{d}{d t}\left(\frac{\partial L}{\partial \dot{q}_{2}}\right)-\frac{\partial L}{\partial q_{2}}+\frac{\partial F}{\partial \dot{q}_{2}}, \\
Q_{2}=-T_{L},
\end{gathered}
$$


the motion equation of mechanical system $j=2$ can be written as

$$
\begin{aligned}
i^{2}\left(J_{1}+J_{e}+\frac{J_{\mathrm{sp}}}{i^{2}}\right) \frac{d \Omega_{2}}{d t}= & -i i_{a} \psi_{f} \sin \theta_{2}-i i_{b} \psi_{f} \sin \left(\theta_{2}-\frac{2}{3} \pi\right) \\
& -i i_{c} \psi_{f} \sin \left(\theta_{2}+\frac{2}{3} \pi\right)-\alpha_{\mathrm{sp}} \theta_{2} \\
& -i^{2}\left(B_{1}+B_{e}+\frac{B_{\mathrm{sp}}}{i^{2}}\right) \Omega_{2}-T_{L} .
\end{aligned}
$$

In terms of $J=J_{1}+J_{e}+\left(J_{\mathrm{sp}} / i^{2}\right), B=B_{1}+B_{e}+\left(B_{\mathrm{sp}} / i^{2}\right)$, that is, (22) can be rewritten as

$$
\begin{aligned}
i^{2} J \frac{d \Omega_{2}}{d t}= & -i i_{a} \psi_{f} \sin \theta_{2}-i i_{b} \psi_{f} \sin \left(\theta_{2}-\frac{2}{3} \pi\right) \\
& -i i_{c} \psi_{f} \sin \left(\theta_{2}+\frac{2}{3} \pi\right)-\alpha_{\mathrm{sp}} \theta_{2}-i^{2} B \Omega_{2}-T_{L} .
\end{aligned}
$$

The electromechanical dynamic model of MEES system in static $\mathrm{ABC}$ coordinates can be obtained through simultaneous equations of (14), (15), (16), (20), and (23).

Define $C_{\mathrm{abc} / \mathrm{dq0}}$ as the transformation matrix from static coordinates of $\mathrm{ABC}$ to rotating frame of $\mathrm{dq} 0$ as follows:

$$
C_{\mathrm{abc} / \mathrm{dq} 0}=\frac{2}{3}\left[\begin{array}{ccc}
\cos \theta & \cos \left(\theta-\frac{2}{3} \pi\right) & \cos \left(\theta+\frac{2}{3} \pi\right) \\
-\sin \theta & -\sin \left(\theta-\frac{2}{3} \pi\right) & -\sin \left(\theta+\frac{2}{3} \pi\right) \\
\frac{1}{2} & \frac{1}{2} & \frac{1}{2}
\end{array}\right]
$$

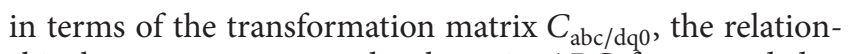
ship between current and voltage in $\mathrm{ABC}$ frame and dq0 frame can be written as

$$
\left[\begin{array}{l}
i_{d} \\
i_{q} \\
i_{0}
\end{array}\right]=C_{\mathrm{abc} / \mathrm{dq} 0}\left[\begin{array}{l}
i_{a} \\
i_{b} \\
i_{c}
\end{array}\right], \quad\left[\begin{array}{l}
u_{d} \\
u_{q} \\
u_{0}
\end{array}\right]=C_{\mathrm{abc} / \mathrm{dq} 0}\left[\begin{array}{l}
u_{a} \\
u_{b} \\
u_{c}
\end{array}\right] .
$$

Substituting (25) into equations (14), (15), and (16), respectively, the voltage equations of the electromagnetic system in dq0 frame can be obtained as

$$
\left[\begin{array}{l}
u_{d} \\
u_{q}
\end{array}\right]=\left[\begin{array}{cc}
L_{d} & 0 \\
0 & L_{q}
\end{array}\right]\left[\begin{array}{l}
\frac{d i_{d}}{d t} \\
\frac{d i_{q}}{d t}
\end{array}\right]+\left[\begin{array}{c}
R_{s} i_{d}-L_{q} \omega_{e} i_{q} \\
R_{s} i_{q}+L_{d} \omega_{e} i_{d}+\omega_{e} \psi_{f}
\end{array}\right]
$$

where $\omega_{e}=p_{n} \Omega_{1}=p_{n} \dot{\theta}_{1}$ is the electric angular speed, $p_{n}$ is the number of pole-pairs, $R_{s}$ is the resistance of the stator, $R_{s}=R_{a}=R_{b}=R_{c}, L_{d}, L_{q}$ are the equivalent inductance of direct axis and quadrature axis, and $L_{d}=L_{q}=L_{s}=3(L-$ $M) / 2$.
Similarly, the motion equations of mechanical system in rotating frame of $\mathrm{dq} 0$ are given as

$$
\begin{aligned}
& {\left[\begin{array}{cc}
J & 0 \\
0 & i^{2} J
\end{array}\right]\left[\begin{array}{c}
\ddot{\theta}_{1} \\
\ddot{\theta}_{2}
\end{array}\right]+\left[\begin{array}{cc}
B & 0 \\
0 & i^{2} B
\end{array}\right]\left[\begin{array}{l}
\dot{\theta}_{1} \\
\dot{\theta}_{2}
\end{array}\right]+\left[\begin{array}{cc}
\frac{\alpha_{\mathrm{sp}}}{i^{2}} & 0 \\
0 & \alpha_{\mathrm{sp}}
\end{array}\right]\left[\begin{array}{l}
\theta_{1} \\
\theta_{2}
\end{array}\right] } \\
&+ {\left[\begin{array}{c}
-\frac{3}{2}\left[i_{q} \psi_{f}+\left(L_{d}-L_{q}\right) i_{d} i_{q}\right] \\
-\frac{3}{2} i\left[i_{q} \psi_{f}+\left(L_{d}-L_{q}\right) i_{d} i_{q}\right]
\end{array}\right]=\left[\begin{array}{l}
-T_{g} \\
-T_{L}
\end{array}\right] . }
\end{aligned}
$$

Due to $\theta_{2}=\theta_{1} / i$ and $T_{g}=T_{L} / i$, two formulas in (27) express the same meaning essentially. Considering $\omega_{e}=p_{n} \Omega_{1}=$ $p_{n} \dot{\theta}_{1}$, namely, $\ddot{\theta}_{1}=\dot{\omega}_{e} / p_{n}$, (27) can be transformed into a new form:

$$
\frac{J}{p_{n}} \dot{\omega}_{e}+\frac{B}{p_{n}} \omega_{e}-\frac{3}{2}\left[i_{q} \psi_{f}+\left(L_{d}-L_{q}\right) i_{d} i_{q}\right]+\frac{\alpha_{\mathrm{sp}}}{i^{2}} \theta_{1}=-T_{g},
$$

The electromechanical dynamic model of MEES system in rotating frame of dq0 can be obtained through simultaneous equations of (26) and (28).

\section{Decoupling Control of the Dynamic Model}

4.1. Fundamental Principle of Direct Feedback Linearization. For an affine nonlinear system in the standard form,

$$
\begin{array}{r}
x=f(x)+\sum_{i=1}^{p} g_{i}(x) u_{i}, \quad y_{i}=h_{i}(x) \\
i=1,2, \ldots, p
\end{array}
$$

where $x \in R^{n}$ are state variables, $u \in R^{p}$ are input variables, $y \in R^{p}$ are output variables, $f, g$ are smooth vectors fields defined on $R^{n}$, and $h$ are smooth scalar functions defined on $R^{n}$. The nonlinearity of MEES dynamic model in rotating frame of dq0 is caused by product terms of $\omega_{e} i_{d}$ and $\omega_{e} i_{q}$, which will weaken the performance of conventional PI controller. Linearization and decoupling control of the system are effective solutions to improve the servo capability. The theory of direct feedback linearization (DFL) is known intimately up to now and is applied widespread to the varieties of nonlinear domains $[18,19]$. The basic principle of DFL is to differentiate the output function repeatedly, until the $r$ th derivatives $y^{(r)}$ of output variable explicitly contain control variable $u$, where $r$ is the relatively degree of the system.

4.2. DFL for MEES System. The state variables $x \in R^{3}$, output variables $y \in R^{2}$, and control variables $u \in R^{2}$ are chosen as $i_{d}, i_{q}, \omega_{e}, i_{d}, \omega_{e}$, and $u_{d}, u_{q}$, respectively. The output variables are differentiated with respect to time until at least an input variable explicitly appears by DFL theory. 
Taking the derivative of $i_{d}$, we can obtain

$$
\dot{i}_{d}=-\frac{R_{s}}{L_{d}} i_{d}+\omega_{e} i_{q}+\frac{1}{L_{d}} u_{d}
$$

(30) contains the input $u_{d}$ and the relatively degree $r_{1}=1$. Introducing a new variable $v_{1}$ makes $\dot{i}_{d}=v_{1}$.

Taking the derivative of $\omega_{e}$, we can obtain

$$
\dot{\omega}_{e}=-\frac{B}{J} \omega_{e}+\frac{3 p_{n}}{2 J} i_{q} \psi_{f}-\frac{p_{n} \alpha_{\mathrm{sp}}}{J i^{2}} \theta_{1}+\frac{p_{n}}{J} T_{g}
$$

as (31) does not contain any input variable, second derivation calculus to $\omega_{e}$ can be obtained as follows:

$$
\begin{aligned}
\ddot{\omega}_{e}= & \frac{B}{J} \dot{\omega}_{e}+\frac{3 p_{n}}{2 J} \dot{i}_{q} \psi_{f}-\frac{p_{n} \alpha_{\mathrm{sp}}}{J i^{2}} \dot{\theta}_{1}+\frac{p_{n}}{J} \dot{T}_{g} \\
= & \frac{B}{J}\left(-\frac{B}{J} \omega_{e}+\frac{3 p_{n}}{2 J} i_{q} \psi_{f}-\frac{p_{n} \alpha_{\mathrm{sp}}}{J i^{2}} \theta_{1}+\frac{p_{n}}{J} T_{g}\right) \\
& +\frac{3 p_{n} \psi_{f}}{2 J}\left[-\frac{1}{L_{q}}\left(R_{s} i_{q}+L_{d} \omega_{e} i_{d}+\omega_{e} \psi_{f}\right)+\frac{1}{L_{q}} u_{q}\right] \\
& -\frac{p_{n} \alpha_{\mathrm{sp}}}{J i^{2}} \frac{\omega_{e}}{p_{n}}+\frac{p_{n}}{J} \dot{T}_{g}
\end{aligned}
$$

(32) contains the input $u_{q}$ and the relatively degree $r_{2}=2$. Introducing another new variable $v_{2}$ makes $\dot{\omega}_{e}=v_{2}$.

The comprehensive order of the system equals $r_{1}+r_{2}=3$, which is exactly equal to the degree of the original system. Hence, a nonlinear state feedback control law is devised as follows:

$$
u=E(x)+D(x) v
$$

where $u=\left[u_{d}, u_{q}\right]^{T}, v=\left[v_{1}, v_{2}\right]^{T}, D(x)=\left[\begin{array}{cc}L_{d} & 0 \\ 0 & 2 J L_{q} / 3 p_{n} \psi_{f}\end{array}\right]$,

$$
\begin{aligned}
& E(x) \\
& \left.=\left[\begin{array}{c}
\frac{2 L_{q} B}{3 p_{n} \psi_{f}}\left(-\frac{B}{J} \omega_{e}+\frac{3 p_{n}}{2 J} i_{q} \psi_{f}-\frac{p_{n} \alpha_{\mathrm{sp}}}{J i^{2}} \theta_{1}+\frac{p_{n}}{J} T_{g}\right) \\
+\left(R_{s} i_{q}+L_{d} \omega_{e} i_{d}+\omega_{e} \psi_{f}\right)+\frac{\alpha_{\mathrm{sp}}}{J i^{2}} \omega_{e}-\frac{p_{n}}{J} \dot{T}_{g}
\end{array}\right]\right] .
\end{aligned}
$$

TABLE 2: Operation parameters of a $0.16 \mathrm{kWh} / 0.8 \mathrm{~kW}$ MEES system.

\begin{tabular}{lc}
\hline Rated work voltage $U_{n}$ & $380 \mathrm{~V}$ \\
Rated work torque & $2.1 \mathrm{~N} \cdot \mathrm{m}$ \\
Rated rotating speed of ES box & $15 \mathrm{r} / \mathrm{min}$ \\
Number of pole pairs for rotor $p_{n}$ & 4 \\
Rated rotating speed of rotor & $600 \mathrm{r} / \mathrm{min}$ \\
Transformation ratio of the gear $i$ & $40: 1$ \\
Moment of inertia for rotor $J_{1}$ & $0.8 \times 10^{-3} \mathrm{~kg} \mathrm{~m}^{2}$ \\
Equivalent moment of inertia for the gear & $0.0013 \mathrm{~kg} \mathrm{~m}{ }^{2}$ \\
$J_{e}$ & \\
Equivalent moment of inertia for the ES & $4.3264 \mathrm{~kg} \mathrm{~m}{ }^{2}$ \\
box $J_{\text {sp }}$ & 5.0178 \\
Torsion constant of spring $\alpha_{\text {sp }}$ & $0.1442 \mathrm{~Wb}$ \\
Effective flux of permanent magnet $\psi_{f}$ & $1.95 \Omega$ \\
Resistance of stator $R_{s}$ & $0.2541 \mathrm{H}$ \\
Equivalent inductance of direct axis $L_{d}$ & $0.2541 \mathrm{H}$ \\
Equivalent inductance of quadrature axis & \\
$L_{q}$ & $0.015 \mathrm{~N} / \mathrm{rad} / \mathrm{s}$ \\
Viscous damping coefficient of the motor & $0.013 \mathrm{~N} / \mathrm{rad} / \mathrm{s}$ \\
$B_{1}$ & $0.005 \mathrm{~N} / \mathrm{rad} / \mathrm{s}$ \\
Viscous damping coefficient of the gear $B_{e}$ & \\
Viscous damping coefficient of the spring & \\
$B_{\text {sp }}$ & \\
\hline
\end{tabular}

Substituting (33) into the electromechanical dynamic model in rotating frame of $\mathrm{dq} 0$, the ultimate linearization equation of the MEES system can be obtained as follows:

$$
\begin{aligned}
& {\left[\begin{array}{c}
i_{d} \\
i_{q} \\
\dot{\omega}_{e}
\end{array}\right]=\left[\begin{array}{ccc}
0 & 0 & 0 \\
0 & \frac{B}{p_{n} J} & -\left(\frac{2 B^{2}}{3 p_{n} \psi_{f} J}+\frac{2 \alpha_{\mathrm{sp}}}{3 \psi_{f} i^{2}}\right) \\
0 & \frac{3 p_{n} \psi_{f}}{2 J} & -\frac{B}{J}
\end{array}\right]\left[\begin{array}{c}
i_{d} \\
i_{q} \\
\omega_{e}
\end{array}\right]} \\
& +\left[\begin{array}{cc}
1 & 0 \\
0 & \frac{2 J}{3 p_{n} \psi_{f}} \\
0 & 0
\end{array}\right]\left[\begin{array}{l}
v_{1} \\
v_{2}
\end{array}\right] \\
& +\left[\begin{array}{c}
0 \\
\frac{2 B}{3 \psi_{f} J} T_{g}+\frac{2}{3 \psi_{f}} \dot{T}_{g}-\frac{2 B p_{n} \alpha_{\mathrm{sp}}}{3 \psi_{f} J i^{2}} \theta_{1} \\
\frac{p_{n}}{J} T_{g}-\frac{p_{n} \alpha_{\mathrm{sp}}}{J i^{2}} \theta_{1}
\end{array}\right] \\
& =A x+E v+C \text {, }
\end{aligned}
$$

where $A, E$, and $C$ are three matrices.

4.3. Design of Optimal Linear Control. Optimal linear control block diagram of speed tracking for linearized system (35) is designed in Figure 3. 


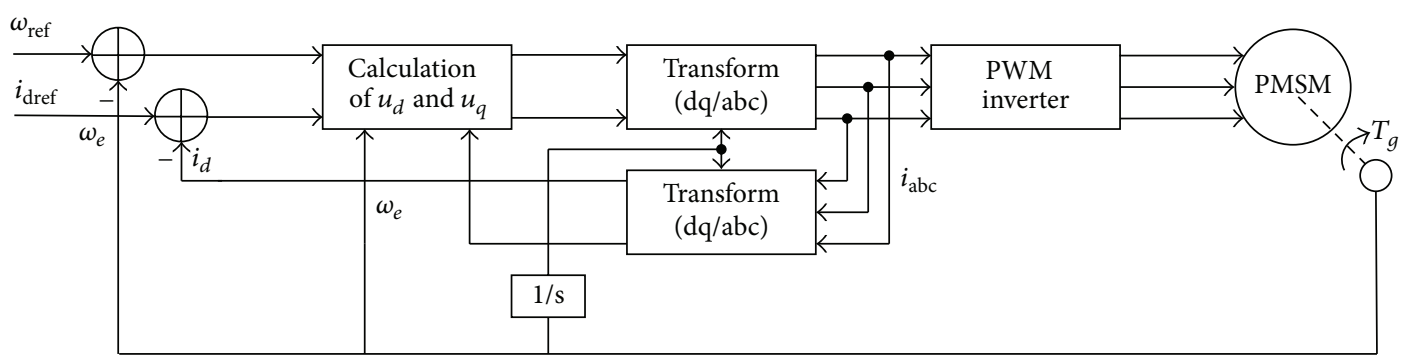

FIGURE 3: Control block diagram of speed tracking for linearized system.

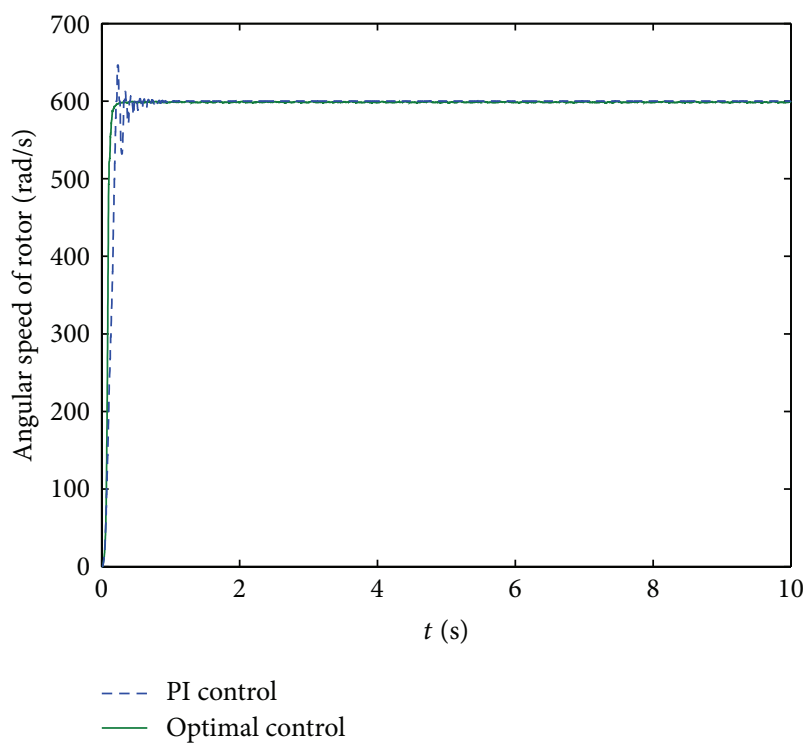

(a) Electric angular speed of rotor

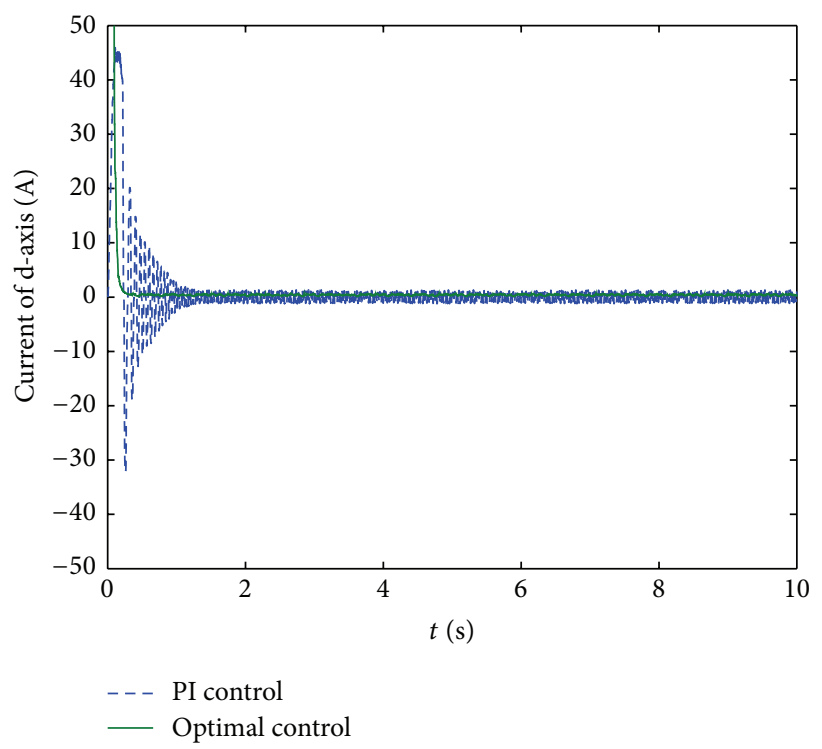

(b) Current of $d$-axis

FIGURE 4: The simulation results of case I (always cutting off the energy storage device).

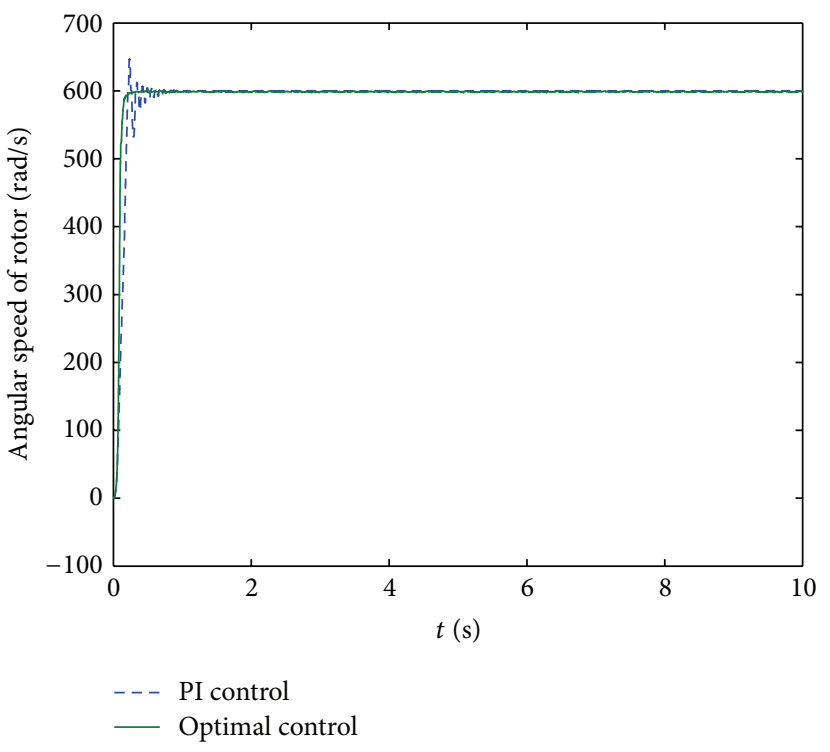

(a) Electric angular speed of rotor

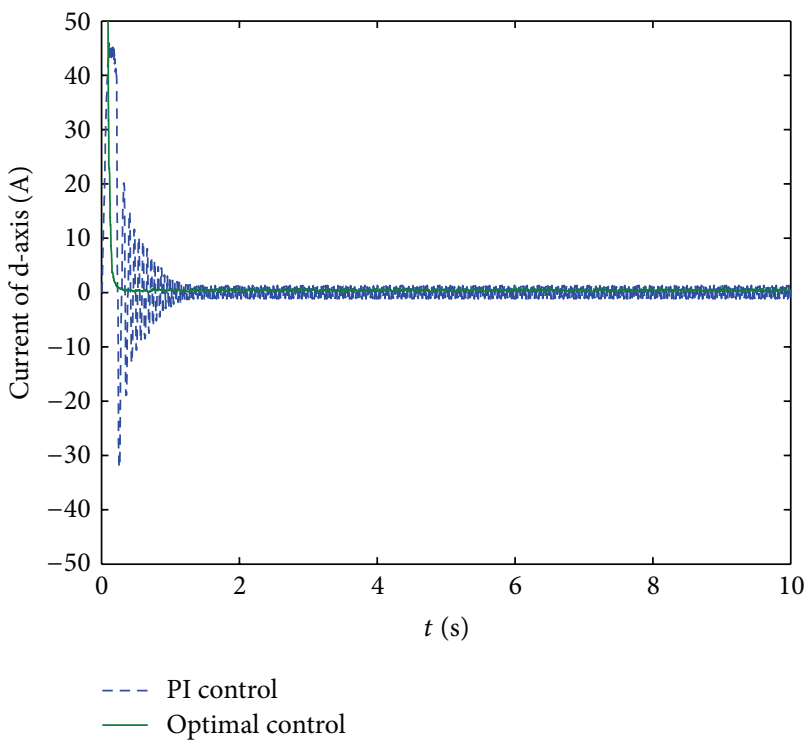

(b) Current of $d$-axis

FIGURE 5: The simulation results of case II (from the initial state of storing no energy to the final state of storing full of energy). 


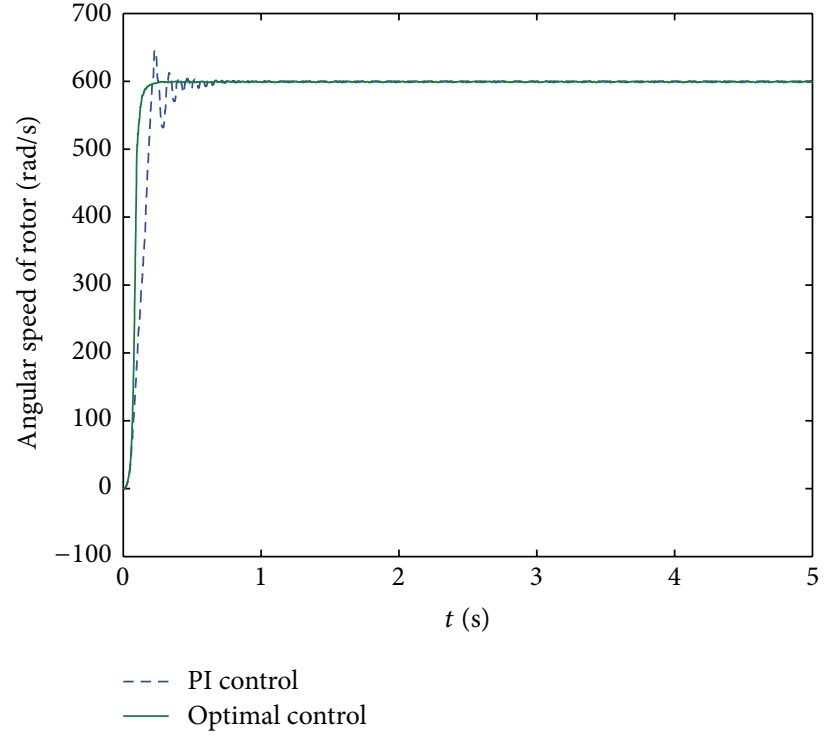

(a) Electric angular speed of rotor

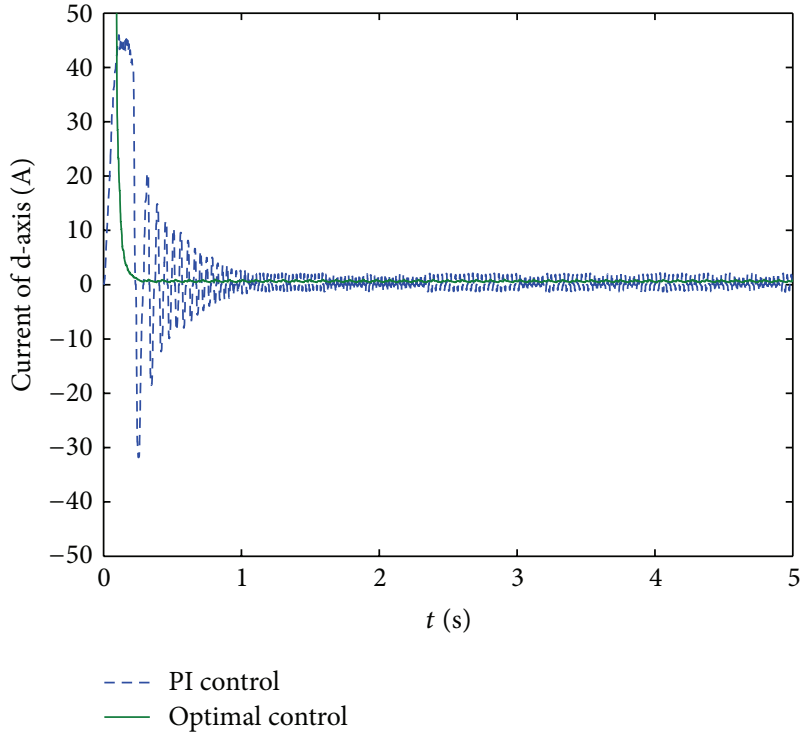

(b) Current of $d$-axis

FIgURE 6: The simulation results of case III (from the initial state of storing half of full of energy to the final state of storing full of energy).

For the linearized system (35), the theory of linear control can be applied to determine $v$. In order to improve the control performance, in terms of optimal control theory of linear system, optimal control inputs are chosen as

$$
v=-K^{*} \cdot x
$$

where $K^{*}=R^{-1} E^{T} P^{*}, R=R^{T}$ is the matrix of weight coefficient.

$P^{*}$ is the solution of Riccati equation as follows:

$$
A^{T} P+P A-P E R^{-1} E^{T} P+Q=0
$$

where $Q$ is a positive definite matrix.

\section{Simulation and Results}

Based on a $0.16 \mathrm{kWh} / 0.8 \mathrm{~kW}$ MEES system being developed, the operation parameters of the system are given in Table 2. In terms of the description above, the control objectives in the paper are determined as the speed of rotor and the current of $d$-axis, namely, the speed of rotor tracking the reference speed and the current of $d$-axis being zero. The reference speed of the motor is set at $600 \mathrm{r} / \mathrm{min}$. The work of modeling and control is implemented in Matlab environment.
In terms of the operation parameters in Table 2, the matrix parameters $A$ and $E$ in (35) are obtained as follows:

$$
\begin{aligned}
A & =\left[\begin{array}{ccc}
0 & 0 & 0 \\
0 & \frac{B}{p_{n} J} & \left(\frac{2 B^{2}}{3 p_{n} \psi_{f} J}+\frac{2 \alpha_{\mathrm{sp}}}{3 \psi_{f} i^{2}}\right) \\
0 & \frac{3 p_{n} \psi_{f}}{2 J} & -\frac{B}{J}
\end{array}\right] \\
& =\left[\begin{array}{ccc}
0 & 0 & 0 \\
0 & 0.0019 & -0.2321 \\
0 & 0.1999 & 0.0076
\end{array}\right] \\
E & =\left[\begin{array}{ccc}
1 & 0 \\
0 & \frac{2 J}{3 p_{n} \psi_{f}} \\
0 & 0
\end{array}\right]=\left[\begin{array}{cc}
1 & 0 \\
0 & 5.0029 \\
0 & 0
\end{array}\right] .
\end{aligned}
$$

In the paper, the matrices of weight coefficient $R$ and positive definite $Q$ are taken as 1 and a $3 \times 3$ identity matrix, respectively. Then, the matrix of $P^{*}$ can be calculated as

$$
P^{*}=\left[\begin{array}{ccc}
1 & 0 & 0 \\
0 & -0.000228 & -0.017318 \\
0 & -4.985561 & -0.000265
\end{array}\right] \text {. }
$$

Considering (36), the inputs of the optimal control can be expressed as

$$
\begin{aligned}
v & =-K^{*} \cdot x=-R^{-1} E^{T} P^{*} \cdot x \\
& =\left[\begin{array}{c}
-i_{d} \\
0.001141 i_{q}+0.086640 \omega_{e}
\end{array}\right] .
\end{aligned}
$$


Substituting (41) into (33), the nonlinear state feedback controller is given as

$$
\begin{aligned}
u= & {\left[\begin{array}{c}
1.6969 i_{d}-0.2541 i_{q} \omega_{e} \\
1.953387 i_{q}+0.265858 \omega_{e}+0.2541 i_{d} \omega_{e}
\end{array}\right] } \\
& +\left[\begin{array}{c}
0 \\
0.008956 T_{g}-0.924108 \dot{T}_{g}-0.000449 \theta_{1}
\end{array}\right] .
\end{aligned}
$$

In order to evaluate the effectiveness of the proposed algorithm properly and adequately, the simulation schemes are selected as three different cases, which are case I (always cutting off the energy storage device), case II (from the initial state of storing no energy to the final state of storing full of energy), and case III (from the initial state of storing half of full of energy to the final state of storing full of energy). The simulation results are shown in Figures 4, 5, and 6, respectively. In order to better display the control performances, the proposed algorithm is in contrast with the conventional PI control. The proportional and integral coefficients for $d$ axis PI current controller are set as 2.6 and 50, respectively, and the proportional and integral coefficients for PI speed tracking controller are set as 0.24 and 10, respectively. Case I simulates PMSM operating in no load state, which is to test the no-load performance of the servomotor. The results in Figure 4 show that the proposed algorithm makes the controlled objective become stable more quickly and almost has no oscillation in stabilization process in comparison with the conventional PI control. Figure 5 shows the results of case II, which essentially represents the process from point $J$ to point $E$ in Figure 2. Figure 6 displays the results of case III, and it should be noted that the running time in the condition will be half of the whole time of energy storage, which is $5 \mathrm{~s}$. The simulation results in case II and case III are similar to case I. Therefore, the simulation results reveal that in contrast with conventional PI control, the presented algorithm not only has a preferable ability of speed tracking in all of the three cases, but also makes the electromechanical system have a better dynamic performance in energy storage process.

\section{Conclusions}

In terms of the characteristics of electromechanical coupling for PMSM based MEES system presented in the paper, dynamic modeling and control of the system are proposed from an electromechanical point of view. The conclusions were made as follows:

(a) dynamic model for PMSM based MEES system is nonlinear with significant electromechanical coupling. The nonlinear model constructed by LagrangeMaxwell energy method can reflect the characteristics of electromechanical coupling for the system;

(b) DFL can perform the dynamic decoupling for the complex electromechanical system completely;

(c) the speed tracking controller proposed in the paper in terms of optimal linear control theory for linearized system has a better performance in contrast with conventional PI control algorithm, and the simulation results in three different cases prove the validity and feasibility of the designed method.

\section{Conflict of Interests}

The authors declare that there is no conflict of interests regarding the publication of this paper.

\section{Acknowledgments}

This research is supported by the Specialized Research Fund for the Doctoral Program of Higher Education of China under Grant no. 20120036130001, the Fundamental Research Funds for the Central Universities of China under Grant no. 11MG40, and the Independent Research Funds of State Key Laboratory of Alternate Electrical Power System with Renewable Energy Sources of China under Grant no. 201209.

\section{References}

[1] K. T. Tan, P. L. So, Y. C. Chu, and M. Z. Q. Chen, "Coordinated control and energy management of distributed generation inverters in a microgrid," IEEE Transactions on Power Delivery, vol. 28, no. 2, pp. 704-713, 2013.

[2] K. T. Tan, X. Y. Peng, P. L. So, Y. C. Chu, and M. Z. Q. Chen, "Centralized control for parallel operation of distributed generation inverters in microgrids," IEEE Transaction on Smart Grid, vol. 3, no. 4, pp. 1977-1987, 2012.

[3] A. Bernstein, D. Bienstock, D. Hay, U. Meric, and Z. Gil, "Sensitivity analysis of the power grid vulnerability to largescale cascading failures," ACM Sigmtrics Performance Evaluation Review, vol. 40, no. 3, pp. 33-37, 2012.

[4] M. Black and G. Strbac, "Value of bulk energy storage for managing wind power fluctuations," IEEE Transactions on Energy Conversion, vol. 22, no. 1, pp. 197-205, 2007.

[5] K. C. Divya and J. Østergaard, "Battery energy storage technology for power systems-an overview," Electric Power Systems Research, vol. 79, no. 4, pp. 511-520, 2009.

[6] A. I. Cohen and S. H. Wan, "An algorithm for scheduling a large pumped storage plant," IEEE Transactions on Power Apparatus System, vol. 104, no. 8, pp. 2099-2104, 1985.

[7] A. J. Pimm, S. D. Garvey, and R. J. Drew, "Shape and cost analysis of pressurized fabric structures for subsea compressed air energy storage," Proceedings of the Institution of Mechanical Engineers C, vol. 225, no. 5, pp. 1027-1043, 2011.

[8] R. Hebner, J. Beno, and A. Walls, "Flywheel batteries come around again," IEEE Spectrum Magazine, vol. 39, no. 4, pp. 4651, 2002.

[9] M. H. Ali, B. Wu, and R. A. Dougal, "An overview of SMES applications in power and energy systems," IEEE Transactions on Sustainable Energy, vol. 1, no. 1, pp. 38-47, 2010.

[10] A. Rufer, D. Hotellier, and P. Barrade, "A supercapacitor-based energy storage substation for voltage compensation in weak transportation networks," IEEE Transactions on Power Delivery, vol. 19, no. 2, pp. 629-636, 2004.

[11] K. Senthil, S. Mitra, A. Roy, A. Sharma, and D. P. Chakravarthy, "Compact inductive energy storage pulse power system," Review of Scientific Instruments, vol. 83, no. 5, Article ID 054703, 4 pages, 2012. 
[12] Y. Yu and Z. Q. Mi, "Nonlinear dynamic model and chaotic characteristics of mechanical elastic energy storage unit in energy storage process," Acta Physica Sinica, vol. 62, no. 3, Article ID 038403, 8 pages, 2013.

[13] Z. Q. Mi, Y. Yu, Z. Q. Wang, and J. Q. Tang, "Preliminary exploration on permanent magnet motor based mechanical elastic energy storage unit and key technical issues," Automation of Electric Power Systems, vol. 37, no. 1, pp. 26-30, 2013.

[14] Y. A. I. Mohamed and T. K. Lee, "Adaptive self-tuning MTPA vector controller for IPMSM drive system," IEEE Transactions on Energy Conversion, vol. 21, no. 3, pp. 636-644, 2006.

[15] S. Jiang and L. Ju, "Study on electromechanical coupling nonlinear vibration of flywheel energy storage system," Science in China, Series E, vol. 49, no. 1, pp. 61-77, 2006.

[16] Z. W. Gao, D. X. Kong, and C. H. Gao, "Modeling and control of complex dynamic systems: applied mathematical aspects," Journal of Applied Mathematics, vol. 2012, Article ID 869792, 5 pages, 2012.

[17] M. J. Mahmoodabadi, A. Bagheri, N. Nariman-Zadeh, A. Jamali, and R. A. Maafi, "Pareto design of decoupled slidingmode controllers for nonlinear systems based on a multiobjective genetic algorithm," Journal of Applied Mathematics, vol. 2012, Article ID 639014, 22 pages, 2012.

[18] G. Kenné, R. Goma, H. Kwawo, F. Lamnabhi-Lagarrigue, A. Arzandé, and J. C. Vannier, "An improved direct feedback linearization technique for transient stability enhancement and voltage regulation of power generators," International Journal of Electrical Power and Energy Systems, vol. 32, no. 7, pp. 809-816, 2010.

[19] Z. H. Abootorabi, G. R. A. Markadeh, and J. Soltani, "Direct torque and flux regulation of synchronous reluctance motor drives based on input-output feedback linearization," Energy Conversion and Management, vol. 51, no. 1, pp. 71-80, 2010. 


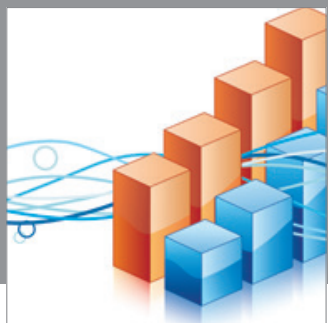

Advances in

Operations Research

mansans

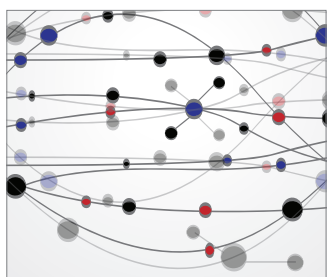

The Scientific World Journal
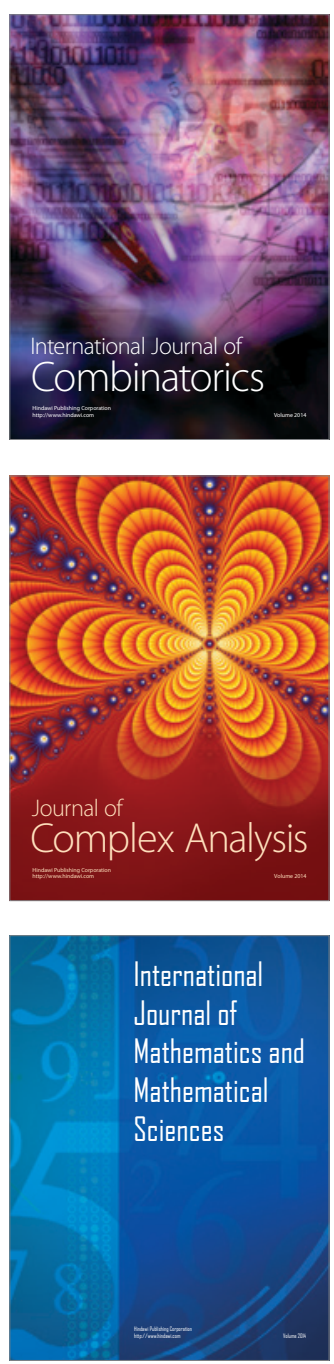
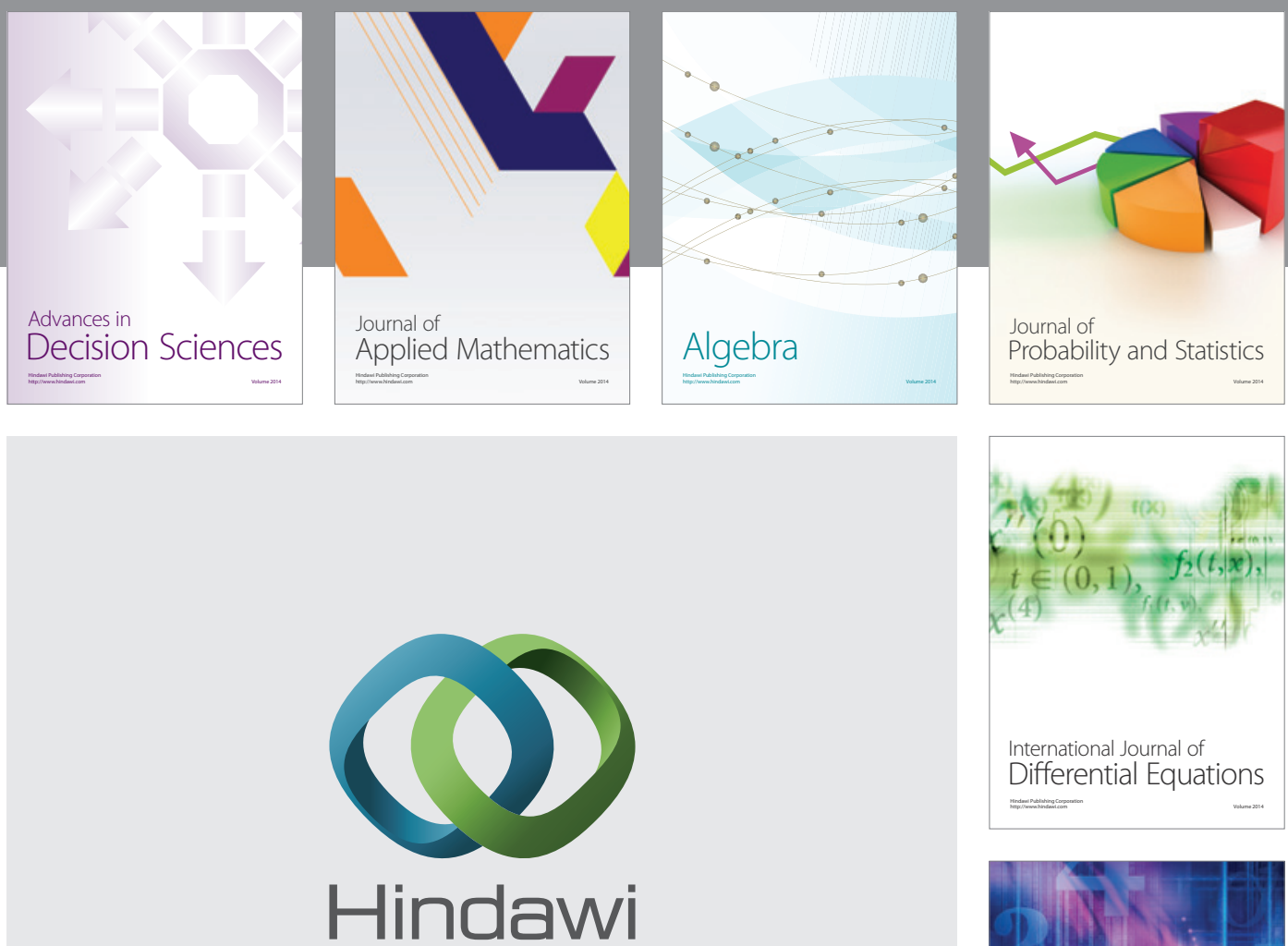

Submit your manuscripts at http://www.hindawi.com
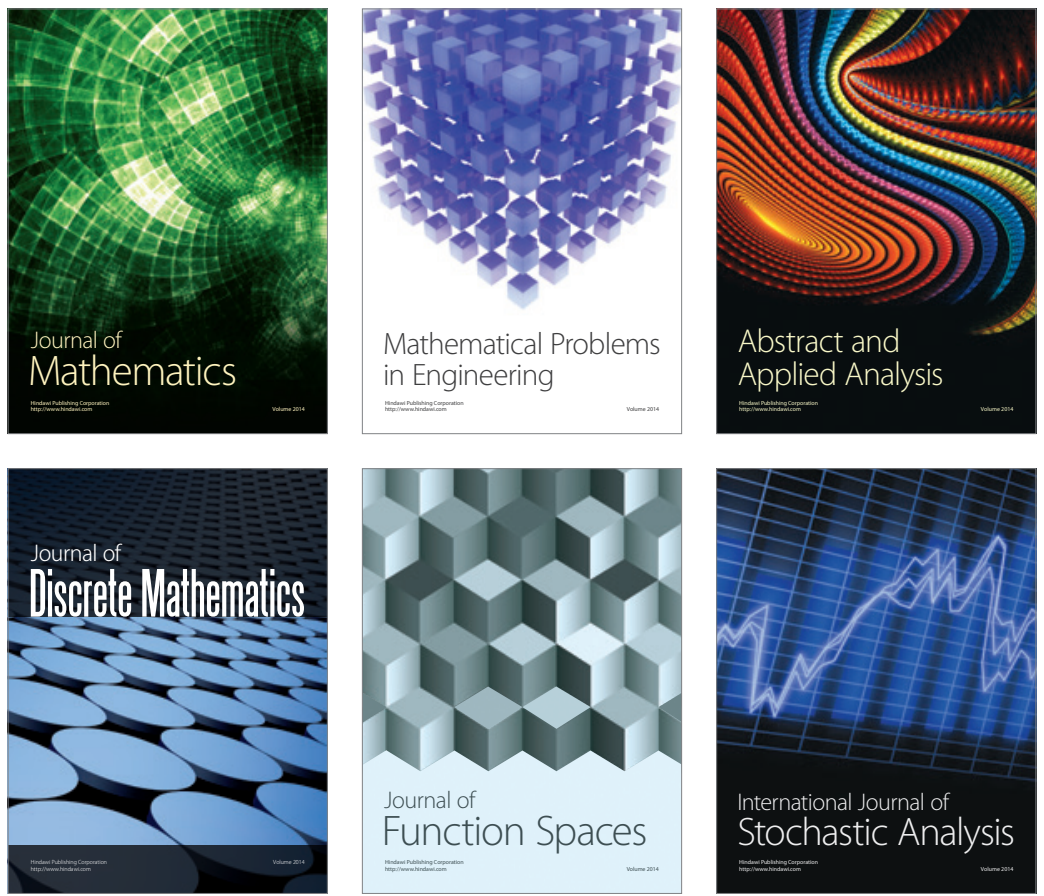

Journal of

Function Spaces

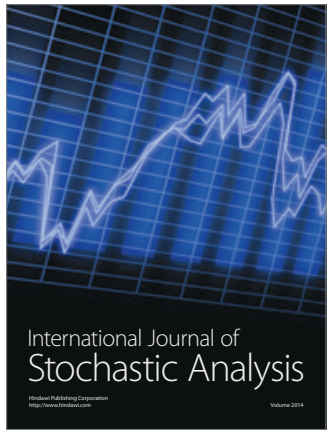

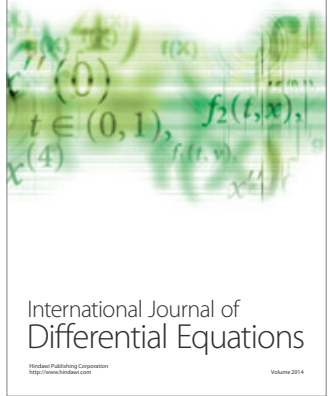
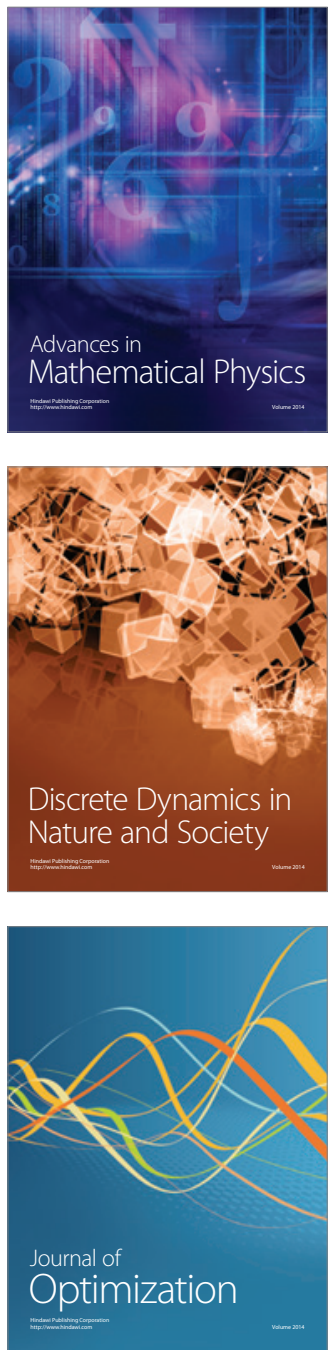